\title{
PARÂMETROS GENÉTICOS E SELEÇÃo DE PROCEDÊNCIAS E PROGÊNIES DE ERVA-MATE NATIVA NO ESTADO DE MATO GROSSO DO SUL
}

\author{
Reginaldo Brito da Costa $^{1}$, Marcos Deon Vilela de Resende ${ }^{2}$, Diego Tyszka Martinez ${ }^{1}$, Antonia \\ Railda Roel ${ }^{3}$, Natasha Brianez Rodrigues ${ }^{1}$, Adriana Zanirato Contini ${ }^{1}$ \\ ${ }^{1}$ Universidade Federal de Mato Grosso, Av. Fernando Corrêa da Costa, no 2367, Bairro Boa Esperança. - 78060-900, Cuiabá, MT. \\ E-mail: reg.brito.costa@gmail.com,diegotyszka@hotmail.com, natashabrianez@gmail.com,dricazc@hotmail.com \\ ${ }^{2}$ Embrapa Florestas, Estrada da Ribeira, km 111, Caixa Postal 319, CEP 83411-000, Colombo, PR. \\ E-mail: marcos.deon@gmail.com \\ ${ }^{3}$ Universidade Católica Dom Bosco, Avenida Tamandaré, no 6000, Jardim Seminário, CEP 79117-900, Campo Grande, MS. \\ E-mail: arroel@ucdb.br
}

\section{RESUMO}

O estudo objetivou estimar parâmetros genéticos e ganho com seleção precoce em procedências e progênies de erva-mate nativas, quanto aos caracteres de crescimento, subsidiando o programa de melhoramento genético da espécie no Estado de Mato Grosso do Sul (Brasil). As sementes foram coletadas de três procedências, com 25 matrizes de polinização aberta de cada procedência, nos municípios de Ponta Porã, Aral Moreira e Laguna Carapã, MS. Os testes foram instalados nos municípios de Tacuru, Amambai e Ponta Porã, sob delineamento de blocos ao acaso com 75 tratamentos e 5 repetições por local. Aos 2 anos de idade, as plantas foram avaliadas quanto aos caracteres altura, diâmetro do coleto e número de lançamentos foliares, usando-se a metodologia de modelo linear misto do software Selegen - Reml/Blup. A maior parte da variação genética encontra-se distribuída dentro das procedências. As herdabilidades individuais no sentido restrito para os caracteres estudados foram de altas magnitudes, indicando expressivo controle genético das progênies e progresso com seleção precoce. O programa de melhoramento genético pode ser conduzido em apenas um dos locais, tendo em vista a similaridade dos ambientes.

Palavras-chave: Ilex paraguariensis, variabilidade genética, ganho genético, modelo linear misto

\section{GENETIC PARAMETERS AND SELECTION OF NATIVE ERVA-MATE PROVENANCES AND PROGENIES IN MATO GROSSO DO SUL STATE}

\begin{abstract}
The objective of this work was to estimate genetic parameters and gain through early selection of native erva-mate provenances and progenies regarding growth parameters and supporting the improvement program of this species in Mato Grosso do Sul State (Brazil). Seeds were collected from three provenances, having 25 open pollination parent trees from each provenance in the Ponta Porã, Aral Moreira and Laguna Carapã municipalities, MS. Trials were carried out in Tacuru, Amambai and Ponta Porã municipalities under a random blocks design with 75 treatments and 5 replications per site. When plants were two years old, they were evaluated for: height, diameter and number of foliar shoots. The mixed linear model method of Selegen - Reml/Blup was used to analyze the data. The greatest part of genetic variation was found within provenance. Strict sense individual heritability for the studied characteristics was of high magnitude, indicating expressive genetic control and progress with early selection. A
\end{abstract}


genetic improvement program can be carried out in only one of the three locations, since all environments were shown to be similar.

Key words: Ilex paraguariensis, genetic variability, genetic gain, linear mixed model

\section{INTRODUÇÃO}

A erva-mate (Ilex paraguariensis $\mathrm{St}$. Hil.) é a espécie mais comum e utilizada do gênero e pertence à família Aquifoliaceae com cerca de 600 espécies. Dessas, 220 são nativas da América do Sul e 68 ocorrem no Brasil, principalmente na região sul (FERREIRA et al., 1983; SCHERER, 1997; STURION \& RESENDE, 2010) e em remanescentes florestais no sul do Estado do Mato Grosso do Sul (POTT \& POTT, 2002). É uma espécie perene bastante apreciada em todo o Brasil na forma de bebidas, insumos para alimentos, produtos de uso e higiene pessoal.

A cultura é uma das melhores opções de geração de emprego e renda, especialmente entre os pequenos e médios produtores rurais. No Brasil a maior parte da produção é extrativista, demonstrando que a atividade ainda está muito dependente dos ervais nativos. Em 2009, no segmento do extrativismo vegetal, a erva-mate nativa ocupou a quarta colocação entre os produtos não madeireiros, com produção equivalente a 218 mil toneladas, o que representa $\mathrm{R} \$$ 86,6 milhões (IBGE, 2010).

As estimativas de parâmetros e ganhos genéticos com seleção têm sido objeto de estudos em testes de progênies para diversas espécies nativas (GONÇALVES et al., 2005 e FURLANI et al., 2005 para a seringueira, FARIAS NETO \& RESENDE, 2001 para a pupunha, CAMARGO et al., 2010 para a castanha-doBrasil, entre outros) e erva-mate (ROSSE \& FERNANDES, 2002 e SIMEÃO et al., 2002), porém ainda são escassas as informações disponíveis na literatura sobre procedências e progênies de erva-mate nativas, relacionadas à seleção precoce do material genético, especialmente no Estado de Mato Grosso do Sul. Os estudos que envolvam a seleção na fase inicial do programa de melhoramento são importantes, constituindo-se em um procedimento eficiente para a maximização do ganho genético (ADAMS et al., 2007), subsidiando o monitoramento das procedências e progênies ao longo do programa de melhoramento.

Nesse sentido, os testes de procedências e progênies são instrumentos importantes para estimação da variância entre procedências, de parâmetros genéticos, seleção de progênies e de indivíduos, com vistas a quantificar e maximizar os ganhos genéticos, além de permitir a utilização de procedimento de seleção adequado.

A utilização da metodologia Reml/Blup, desenvolvida para o melhoramento de plantas perenes tem maximizado os ganhos genéticos com seleção (RESENDE, 2002b; MISSIO et al., 2005), por tratar-se de um procedimento estimativo, especialmente para dados desbalanceados, predizendo valores genéticos em testes de procedências e progênies.

O presente estudo objetivou estimar parâmetros genéticos e ganho com seleção precoce em procedências e progênies de erva-mate, quanto aos caracteres de crescimento de plantas nativas, subsidiando o programa de melhoramento da espécie, no Estado de Mato Grosso do Sul.

\section{MATERIAL E MÉTODOS}

As sementes de erva-mate foram coletadas nos municípios de Ponta Porã $\left(23^{\circ} 32^{\prime} 30^{\prime \prime} \mathrm{S}\right.$ e $\left.5^{\circ} 37^{\prime} 30^{\prime \prime} \mathrm{W}\right)$, Aral Moreira $\left(22^{\circ} 56^{\prime} 02^{\prime \prime} \mathrm{S}\right.$ e $\left.55^{\circ} 38^{\prime} 07^{\prime \prime} \mathrm{W}\right)$ e Laguna 


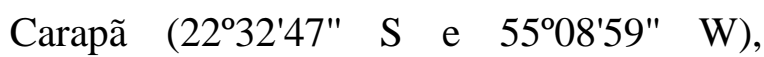
localizados em área de fronteira entre Brasil e Paraguai, na região sul do Estado de Mato Grosso do Sul, as quais foram denominadas procedências. De cada procedência, foram coletadas sementes de 25 matrizes nativas de polinização aberta.

$\mathrm{O}$ testes de procedências e progênies foram instalados nos municípios de Tacuru, Amambai e Ponta Porã, no estado de Mato Grosso do Sul (Tabela 1), em agosto de 2009, sob delineamento de blocos ao acaso com 75 tratamentos (25 progênies de cada local), 5 repetições e 10 plantas por parcela em linhas simples, no espaçamento $3 \mathrm{~m} \mathrm{x} 2$ m. O preparo do solo foi realizado utilizando-se aração e gradagem, com limpezas e controle manual de plantas invasoras após o estabelecimento do experimento.
Aos 2 anos de idade, as progênies foram avaliadas quanto aos caracteres: altura total das mudas expressas em centímetros (ALT); diâmetro do coleto expresso em milímetros (DC) e, número de lançamentos foliares (NF).

As variáveis foram analisadas usandose a metodologia de modelo linear misto univariado aditivo do software Selegen Reml/Blup (restricted maximum likelihoodbest linear unbiased prediction) apresentado por Resende (2002a), cujo modelo consistiu do seguinte:

$\mathrm{y}=\mathrm{Xb}+\mathrm{Za}+\mathrm{Wc}_{1}+\mathrm{Qr}+\mathrm{Uc}_{2}+\mathrm{e}$, em que: $\mathrm{y}, \mathrm{b}, \mathrm{a}, \mathrm{c}_{1}, \mathrm{r}, \mathrm{c}_{2}$, e: vetores de dados, dos efeitos de repetição somados à média geral, dos efeitos genéticos aditivos individuais, dos efeitos de parcela, dos efeitos de população ou procedência, dos efeitos da interação genótipos $\mathrm{x}$ ambientes e de erros ou resíduos respectivamente.

Tabela 1. Detalhamento dos municípios de coleta dos dados das procedências e progênies nos municípios de Tacuru, Amambai e Ponta Porã, no Estado de Mato Grosso do Sul.

\begin{tabular}{l|c|c|c}
\hline Variáveis Descritivas & \multicolumn{3}{|c}{ Municípios } \\
\cline { 2 - 4 } & Tacuru & Amambai & Ponta Porã \\
\hline $\begin{array}{l}\text { Altitude (m) } \\
\text { Classificação } \\
\text { climática de Köppen }\end{array}$ & 372 & 480 & 755 \\
$\begin{array}{l}\text { Umidade relativa } \\
\text { média (\%) }\end{array}$ & $\mathrm{Aw}$ & $\mathrm{Aw}$ & $\mathrm{Aw}$ \\
$\begin{array}{l}\text { Latitude } \\
\text { Longitude }\end{array}$ & 60 & 60 & 60 \\
$\begin{array}{l}\text { Temperatura média } \\
\text { anual }\left({ }^{\circ} \mathrm{C}\right)\end{array}$ & $23^{\circ} 37^{\prime} 57^{\prime \prime} \mathrm{S}$ & $23^{\circ} 06^{\prime} 15^{\prime \prime} \mathrm{S}$ & $23^{\circ} 32^{\prime} 30^{\prime \prime} \mathrm{S}$ \\
$\begin{array}{l}\text { Precipitação média } \\
\text { anual (mm) }\end{array}$ & $55^{\circ} 00^{\prime} 57^{\prime \prime} \mathrm{W}$ & $55^{\circ} 13^{\prime} 33^{\prime \prime} \mathrm{W}$ & $55^{\circ} 37^{\prime} 30^{\prime \prime} \mathrm{W}$ \\
& 21 & 22 & 20 \\
Classe de solo & 1.500 & 1.400 & 1.660 \\
& $\begin{array}{c}\text { Neossolo } \\
\text { Muartzarênico com } \\
\text { manchas de }\end{array}$ & Latossolo Vermelho & Latossolo Vermelho \\
\hline
\end{tabular}


X, Z, W, Q e U: matrizes de incidência para os respectivos efeitos.

As equações de modelo misto foram:

$\left[\begin{array}{ccccc}X^{\prime} X & X^{\prime} Z & X^{\prime} W & X^{\prime} Q & X^{\prime} U \\ Z^{\prime} X & Z^{\prime} Z+A^{-1} \lambda_{1} & Z^{\prime} W & Z^{\prime} Q & Z^{\prime} U \\ W^{\prime} X & W^{\prime} Z & W^{\prime} W+I \lambda_{2} & W^{\prime} Q & W^{\prime} U \\ Q^{\prime} X & Q^{\prime} Z & Q^{\prime} W & Q^{\prime} Q+I \lambda_{3} & Q^{\prime} U \\ U^{\prime} X & U^{\prime} Z & U^{\prime} W & U^{\prime} Q & U^{\prime} U+I \lambda_{4}\end{array}\right]\left[\begin{array}{c}\hat{b} \\ \hat{a} \\ \hat{c}_{1} \\ \hat{r} \\ \hat{c}_{2}\end{array}\right]=\left[\begin{array}{c}X^{\prime} y \\ Z^{\prime} y \\ W^{\prime} y \\ Q^{\prime} y \\ U^{\prime} y\end{array}\right]$, em que:

$\lambda_{1}=\frac{\sigma_{\mathrm{e}}^{2}}{\sigma_{\mathrm{a}}^{2}}=\frac{1-\mathrm{h}^{2}-\mathrm{c}_{1}^{2}-\mathrm{r}^{2}-\mathrm{c}_{2}^{2}}{\mathrm{~h}^{2}}$;

$\lambda_{2}=\frac{\sigma_{\mathrm{e}}^{2}}{\sigma_{\mathrm{c}_{1}}^{2}}=\frac{1-\mathrm{h}^{2}-\mathrm{c}_{1}^{2}-\mathrm{r}^{2}-\mathrm{c}_{2}^{2}}{\mathrm{c}_{1}^{2}}$

$\lambda_{3}=\frac{\sigma_{\mathrm{e}}^{2}}{\sigma_{\mathrm{r}}^{2}}=\frac{1-\mathrm{h}^{2}-\mathrm{c}_{1}^{2}-\mathrm{r}^{2}-\mathrm{c}_{2}^{2}}{\mathrm{r}^{2}}$

$\lambda_{4}=\frac{\sigma_{\mathrm{e}}^{2}}{\sigma_{\mathrm{c}_{2}}^{2}}=\frac{1-\mathrm{h}^{2}-\mathrm{c}_{1}^{2}-\mathrm{r}^{2}-\mathrm{c}_{2}^{2}}{\mathrm{c}^{2}}$.

$\mathrm{h}^{2}=\frac{\sigma_{\mathrm{a}}^{2}}{\sigma_{\mathrm{a}}^{2}+\sigma_{\mathrm{c}_{1}}^{2}+\sigma_{\mathrm{r}}^{2}+\sigma_{\mathrm{c}_{2}}^{2}+\sigma_{\mathrm{e}}^{2}}$, herdabilidade individual no sentido restrito.

$c_{1}^{2}=\frac{\sigma_{c_{1}}^{2}}{\sigma_{\mathrm{a}}^{2}+\sigma_{\mathrm{c}_{1}}^{2}+\sigma_{\mathrm{r}}^{2}+\sigma_{\mathrm{c}_{2}}^{2}+\sigma_{\mathrm{e}}^{2}}$ : coeficiente de determinação dos efeitos da parcela.

$\mathrm{r}^{2}=\frac{\sigma_{\mathrm{r}}^{2}}{\sigma_{\mathrm{a}}^{2}+\sigma_{\mathrm{c}_{1}}^{2}+\sigma_{\mathrm{r}}^{2}+\sigma_{\mathrm{c}_{2}}^{2}+\sigma_{\mathrm{e}}^{2}}$ : coeficiente de determinação dos efeitos de procedências ou

populações.

$\mathrm{c}_{2}^{2}=\frac{\sigma_{\mathrm{c}_{2}}^{2}}{\sigma_{\mathrm{a}}^{2}+\sigma_{\mathrm{c}_{1}}^{2}+\sigma_{\mathrm{r}}^{2}+\sigma_{\mathrm{c}_{2}}^{2}+\sigma_{\mathrm{e}}^{2}}$ : coeficiente de determinação dos efeitos da interação progênies $\mathrm{x}$ ambientes.

O estimador iterativo dos componentes de variância por REML via algoritmo EM foram:

$$
\begin{aligned}
& \hat{\sigma}_{\mathrm{e}}^{2}=\left[\mathrm{y}^{\prime} \mathrm{y}-\hat{\mathrm{b}}^{\prime} \mathrm{X}^{\prime} \mathrm{y}-\hat{\mathrm{a}}^{\prime} \mathrm{Z}^{\prime} \mathrm{y}-\hat{\mathrm{c}}_{1}^{\prime} \mathrm{W}^{\prime} \mathrm{y}-\hat{\mathrm{r}}^{\prime} \mathrm{Q}^{\prime} \mathrm{y}-\hat{\mathrm{c}}_{2}^{\prime} \mathrm{U}^{\prime} \mathrm{y}\right] /[\mathrm{N}-\mathrm{r}(\mathrm{x})] \\
& \hat{\sigma}_{\mathrm{a}}^{2}=\left[\hat{a}^{\prime} \mathrm{A}^{-1} \hat{\mathrm{a}}+\hat{\sigma}_{\mathrm{e}}^{2} \operatorname{tr}\left(\mathrm{A}^{-1} \mathrm{C}^{22}\right)\right] / \mathrm{q} \\
& \hat{\sigma}_{\mathrm{c}_{1}}^{2}=\left[\hat{\mathrm{c}}_{1}^{\prime} \hat{\mathrm{c}}_{1}+\hat{\sigma}_{\mathrm{e}}^{2} \operatorname{tr} \mathrm{C}^{33}\right] / \mathrm{s}_{1} \\
& \hat{\sigma}_{\mathrm{r}}^{2}=\left[\hat{\mathrm{r}}^{\prime} \hat{\mathrm{r}}+\hat{\sigma}_{\mathrm{e}}^{2} \operatorname{tr} \mathrm{C}^{44}\right] / \mathrm{t} \\
& \hat{\sigma}_{\mathrm{c}_{2}}^{2}=\left[\hat{\mathrm{c}}_{2} \hat{\mathrm{c}}_{2}+\hat{\sigma}_{\mathrm{e}}^{2} \operatorname{tr} \mathrm{C}^{55}\right] / \mathrm{s}_{2}, \text { em que: }
\end{aligned}
$$


$\mathrm{q}, \mathrm{s}_{1}, \mathrm{t}$ e $\mathrm{s}_{2}$, número de indivíduos, de parcelas, de procedências e de combinações progênies $\mathrm{x}$ ambientes, respectivamente.

$\hat{\sigma}_{\mathrm{a}}^{2}$, variância genética aditiva livre da interação $\mathrm{G} \times \mathrm{E}$;

$\hat{\sigma}_{\mathrm{r}}^{2}$, variância genotípica entre procedências;

$\hat{\sigma}_{\mathrm{cl}}^{2}$, variância entre parcelas;

$\hat{\sigma}_{\mathrm{c} 2}^{2}$, variância da interação progênies $\mathrm{x}$ ambientes

$\mathrm{CV}_{\text {gi }}(\%)=\frac{\sqrt{\hat{\sigma}_{\mathrm{a}}^{2}}}{\overline{\mathrm{X}}} \cdot 100$

$\hat{\sigma}_{\mathrm{e}}^{2}$, variância residual dentro da parcela (ambiental + não aditiva);

$\mathrm{r}_{\mathrm{gloc}}=\frac{\hat{\sigma}_{\mathrm{a}}^{2}}{\hat{\sigma}_{\mathrm{a}}^{2}+4 \hat{\sigma}_{\mathrm{c} 2}^{2}}$, correlação genotípica entre o desempenho das progênies nos vários locais;

\section{RESULTADOS E DISCUSSÃO}

As herdabilidades individuais no sentido restrito para os caracteres estudados foram altas, o que demonstra expressivo controle genético e, conseqüentemente, possibilidades de sucesso com a seleção (Tabela 2). Valores moderados de herdabilidade individual para caracteres de crescimento em populações autóctones de erva-mate foram encontrados por Costa et al. (2009) no Estado de Mato Grosso do Sul e valores moderados e altos na região sul do país (ROSSE \& FERNANDES, 2002; SIMEÃO et al., 2002).

As correlações genotípicas através dos locais relacionam o desenvolvimento e o comportamento das progênies em diferentes ambientes. As correlações genotípicas das progênies nos três ambientes foram altas para os três caracteres avaliados, indicando que a classificação das progênies através dos ambientes é semelhante. Estes resultados são corroborados pelos baixos valores da variância da interação progênies $\mathrm{x}$ ambientes que, da mesma forma, indicam maior similaridade do desempenho dos genótipos nos três ambientes. Os resultados demonstram que uma única estratégia de melhoramento por característica poderá ser adotada em apenas um dos locais, embora a altitude do município de Ponta Porã seja maior que a dos municípios de Tacuru e Amambai.

A magnitude dos coeficientes de variação genética individual (CVgi\%), considerando a idade precoce que os genótipos foram avaliados, sugerem boas perspectivas para a seqüência do programa de melhoramento para o caráter número de lançamentos foliares. Os dados obtidos são condizentes àqueles encontrados por Costa et al., (2005). A idade avaliada de forma precoce pode ter contribuído na baixa expressão genética dos caracteres diâmetro e altura nesta fase do desenvolvimento das plantas. s variâncias estimadas para o efeito de procedências demonstram que há pouca variabilidade genética entre elas, sendo de $3,38 \%$ para altura, 2,50\% para diâmetro e $7,05 \%$ para número de lançamentos foliares.

A estimativa dos efeitos genéticos e do ganho genético com a seleção (Tabela 3) apresentou pouca variação entre procedências, o que pode ter sido causado pela proximidade dos locais de coleta ou ainda pela possibilidade de serem populações com certo grau de endogamia. Apesar disso, destacou-se a procedência Ponta Porã, com maior diâmetro e número de lançamentos foliares, na média geral. Neste caso, a maior variabilidade genética 
está dentro de procedências, o que não compensaria esforços futuros na coleta destas fontes de sementes, considerando-se os três locais como uma única procedência. A continuidade do monitoramento da variabilidade genética em idades mais avançadas oferecerá subsídios para delinear estratégias a serem adotadas ao longo do programa de melhoramento genético da erva-mate nativa do Estado de Mato Grosso do Sul.

Em relação aos locais de estudo, através dos valores genéticos preditos, nota- se a predominância de melhores desempenhos das progênies em Ponta Porã para os caracteres diâmetro e número de lançamentos foliares (Tabela 4). Para o caráter altura, Amambai apresentou resultados expressivos, especialmente para a progênie 25. Simeão et al., (2002) enfatizam que os valores genéticos preditos em relação a todos os indivíduos candidatos possibilitam estabelecer a melhor estratégia para o aumento da eficiência do melhoramento.

Tabela 2. Análise conjunta dos três municípios (Tacuru, Amambai e Ponta Porã) para procedências e progênies, referentes aos caracteres altura - ALT $(\mathrm{cm})$, diâmetro - DC (mm), e no de lançamentos foliares - NF em erva-mate, aos 2 anos de idade, no Estado de Mato Grosso do Sul.

\begin{tabular}{c|c|c|c}
\hline \multirow{2}{*}{ Estimativas $^{1}$} & \multicolumn{3}{|c}{ Caracteres } \\
\cline { 2 - 4 } & ALT & DC & $0,38 \pm 0,09$ \\
\hline$\hat{\mathrm{h}}^{2}$ & $0,53 \pm 0,11$ & $0,44 \pm 0,10$ & 0,59 \\
\hline$\hat{\sigma}_{\mathrm{a}}^{2}$ & 0,31 & 0,03 & 0,07 \\
\hline$\hat{\sigma}_{\mathrm{c} 1}^{2}$ & 0,07 & 0,11 & 0,70 \\
\hline$\hat{\sigma}_{\mathrm{e}}^{2}$ & 0,18 & 0,02 & 1,56 \\
\hline$\hat{\sigma}_{\mathrm{f}}^{2}$ & 0,59 & 0,16 & 0,11 \\
\hline$\hat{\sigma}_{\mathrm{r}}^{2}$ & 0,02 & 0,004 & 0,017 \\
\hline$\hat{\sigma}_{\mathrm{c} 2}^{2}$ & 0,007 & 0,0008 & 0,77 \\
\hline $\mathrm{r}_{\mathrm{gloc}}$ & 0,70 & 0,75 & 12,23 \\
\hline Média geral & 34,59 & 20,49 & 6,28 \\
\hline $\mathrm{CV}$ & 1,62 & 0,88 & $\mathrm{~g}$ \\
\hline gi & & & \\
\hline
\end{tabular}

1 Herdabilidade individual no sentido restrito no bloco ( ), variância genética aditiva ( ), variância ambiental entre parcelas ( ), variância residual dentro de parcela (ambiental + não aditiva ( ), variância fenotípica individual ( ), variância de procedência ( ), variância da interação progênies x ambientes ( ), correlação genotípica através dos locais ( ), média geral e coeficiente de variação genética individual (CVgi\%). 
Tabela 3. Efeito genético aditivo $(\mathrm{g})$, valores genéticos $(\mathrm{u}+\mathrm{g})$, ganho e nova média das procedências, na análise conjunta dos três municípios (Tacuru, Amambai e Ponta Porã), quanto aos caracteres altura, diâmetro, e número de lançamentos foliares, no Estado de Mato Grosso do Sul.

\begin{tabular}{c|c|c|c|c|c}
\hline \multirow{2}{*}{ Caráter } & Procedências & $\begin{array}{c}\text { Efeito } \\
\text { Genético } \\
\text { Aditivo }(\mathrm{g})\end{array}$ & $\begin{array}{c}\text { Valores } \\
\text { Genéticos } \\
(\mathrm{u}+\mathrm{g})\end{array}$ & $\begin{array}{c}\text { Ganho } \\
\text { genético }\end{array}$ & $\begin{array}{c}\text { Nova Média } \\
\text { da População }\end{array}$ \\
\hline \multirow{2}{*}{$\begin{array}{c}\text { Altura } \\
(\mathrm{cm})\end{array}$} & Ponta Porã & 0,072 & 34,665 & 0,072 & 34,665 \\
\cline { 2 - 5 } & Laguna Carapã & 0,060 & 34,650 & 0,066 & 34,657 \\
\cline { 2 - 5 } & Aral Moreira & $-0,144$ & 34,445 & 0,000 & 34,593 \\
\hline \multirow{2}{*}{$\begin{array}{c}\text { Diâmetro } \\
(\mathrm{mm})\end{array}$} & Ponta Porã & 0,062 & 20,552 & 0,062 & 20,552 \\
\cline { 2 - 6 } & Laguna Carapã & 0,0006 & 20,490 & 0,031 & 20,521 \\
\cline { 2 - 6 } & Aral Moreira & $-0,063$ & 20,427 & 0,000 & 20,490 \\
\hline \multirow{2}{*}{$\begin{array}{c}\text { Número de } \\
\text { Lançamentos } \\
\text { Foliares }\end{array}$} & Ponta Porã & 0,3409 & 12,570 & 0,341 & 12,569 \\
\cline { 2 - 6 } & Laguna Carapã & 0,096 & 12,3236 & 0,218 & 12,446 \\
\cline { 2 - 6 } & Aral Moreira & $-0,436$ & 11,791 & 0,000 & 12,228 \\
\hline
\end{tabular}

Tabela 4. Melhores progênies, para os caracteres altura $(\mathrm{cm})$, diâmetro $(\mathrm{mm})$ e número de lançamentos foliares de erva-mate, aos 2 anos de idade, nos municípios de Tacuru (1), Amambai (2) e Ponta Porã (3), no Estado de Mato Grosso do Sul.

\begin{tabular}{|c|c|c|c|c|c|c|c|c|}
\hline Bloco & Local & Progênie & Árvore & $\begin{array}{c}\text { Valores } \\
\text { fenotípicos }\end{array}$ & $\begin{array}{c}\text { Valores } \\
\text { genéticos } \\
(\mathrm{u}+\mathrm{a})\end{array}$ & $\begin{array}{c}\text { Ganho } \\
\text { genético } \\
(\%)\end{array}$ & $\begin{array}{l}\text { Nova } \\
\text { Média }\end{array}$ & $\begin{array}{l}\text { Acurácia } \\
\text { Seletiva }\end{array}$ \\
\hline \multicolumn{9}{|c|}{ Altura $(\mathrm{cm})$} \\
\hline 1 & 2 & 25 & 1 & 37,300 & 36,087 & 4,328 & 36,087 & \\
\hline 3 & 3 & 18 & 2 & 37,100 & 36,041 & 4,261 & 36,064 & \\
\hline 1 & 3 & 4 & 1 & 37,500 & 35,844 & 4,050 & 35,991 & \\
\hline 1 & 2 & 10 & 3 & 37,300 & 35,821 & 3,926 & 35,948 & \\
\hline 5 & 1 & 4 & 1 & 36,400 & 35,801 & 3,848 & 35,921 & \\
\hline 5 & 2 & 24 & 3 & 36,400 & 35,811 & 3,790 & 35,901 & \\
\hline 2 & 2 & 25 & 1 & 36,400 & 35,779 & 3,738 & 35,883 & \\
\hline 1 & 3 & 16 & 3 & 37,000 & 35,778 & 3,700 & 35,870 & \\
\hline 2 & 2 & 21 & 3 & 36,700 & 35,745 & 3,660 & 35,856 & \\
\hline 4 & 2 & 13 & 4 & 36,400 & 35,740 & 3,602 & 35,836 & 0,81 \\
\hline \multicolumn{9}{|c|}{ Diâmetro (mm) } \\
\hline 1 & 3 & 16 & 2 & 23,180 & 21,627 & 5,549 & 21,627 & \\
\hline 1 & 3 & 4 & 1 & 21,780 & 21,012 & 4,051 & 21,320 & \\
\hline 3 & 3 & 21 & 2 & 21,180 & 20,905 & 3,377 & 21,182 & \\
\hline 2 & 3 & 21 & 1 & 21,180 & 20,893 & 3,021 & 21,109 & \\
\hline 4 & 3 & 4 & 2 & 21,280 & 20,875 & 2,796 & 21,063 & \\
\hline 4 & 3 & 21 & 4 & 21,080 & 20,868 & 2,635 & 21,030 & \\
\hline 3 & 3 & 21 & 1 & 21,080 & 20,859 & 2,518 & 21,006 & \\
\hline 4 & 3 & 6 & 2 & 21,180 & 20,856 & 2,426 & 20,987 & \\
\hline 5 & 3 & 19 & 1 & 21,180 & 20,854 & 2,352 & 20,972 & \\
\hline 4 & 3 & 18 & 3 & 21,180 & 20,853 & 2,294 & 20,960 & 0,79 \\
\hline
\end{tabular}


Continuação...

\begin{tabular}{ccccccccc}
\hline Bloco & Local & Progênie & Árvore & $\begin{array}{c}\text { Valores } \\
\text { fenotípicos }\end{array}$ & $\begin{array}{c}\text { Valores } \\
\text { genéticos } \\
(\mathrm{u}+\mathrm{a})\end{array}$ & $\begin{array}{c}\text { Ganho } \\
\text { genético } \\
(\%)\end{array}$ & $\begin{array}{c}\text { Nova } \\
\text { Média }\end{array}$ & $\begin{array}{c}\text { Acurácia } \\
\text { Seletiva }\end{array}$ \\
\hline \multicolumn{7}{c}{ Número de lançamentos foliares } \\
\hline 5 & 3 & 9 & 1 & 18,000 & 14,882 & 21,709 & 14,885 & \\
5 & 2 & 24 & 1 & 17,000 & 14,249 & 19,133 & 14,570 & \\
3 & 3 & 24 & 1 & 16,000 & 14,170 & 18,038 & 14,436 & \\
1 & 3 & 23 & 4 & 16,000 & 14,155 & 17,465 & 14,366 & \\
1 & 3 & 9 & 1 & 16,000 & 14,060 & 16,975 & 14,306 & \\
2 & 3 & 23 & 1 & 16,000 & 14,040 & 16,378 & 14,233 & \\
2 & 3 & 23 & 4 & 16,000 & 14,040 & 16,182 & 14,209 & \\
3 & 3 & 18 & 4 & 16,000 & 14,000 & 16,075 & 14,196 & \\
4 & 3 & 7 & 1 & 16,000 & 13,964 & 15,814 & 14,164 & 0,79 \\
\hline
\end{tabular}

Os ganhos genéticos estimados com a seleção dos melhores indivíduos para os caracteres variaram de 3,60 a 4,23\% para altura, 2,29 a 5,55\% para diâmetro e 15,81 a $21,71 \%$ para número de lançamentos foliares. A seleção dos 10 melhores indivíduos elevou a nova média da população para, no mínimo, de $34,59 \mathrm{~cm}$ a $35,83 \mathrm{~cm}$, de $20,49 \mathrm{~mm}$ a $20,96 \mathrm{~mm}$ e de 12,23 a 14,16 para altura, diâmetro e número de lançamentos foliares, respectivamente.

Os valores de acurácia seletiva correspondentes aos três caracteres analisados foram superiores a 79\%. Isto, associado às elevadas correlações genotípicas através dos locais reforçam uma condição muito favorável para o programa de melhoramento genético da erva-mate na região.

\section{CONCLUSÕES}

As herdabilidades individuais obtidas demonstram expressivo controle genético a ser explorado na seleção.

A maior parte da variação genética encontra-se distribuída dentro das procedências.

A seleção precoce dos melhores genótipos proporciona ganhos substanciais para o caráter número de lançamentos foliares.

O programa de melhoramento genético pode ser conduzido em apenas um dos locais, tendo em vista a similaridade dos ambientes.

\section{AGRADECIMENTOS}

Os autores agradecem à Empresa ervateira Santo Antônio pelo apoio logístico durante o estabelecimento do experimento nas fases de viveiro e campo, bem como ao $\mathrm{CNPq}$ pelas bolsas concedidas.

\section{REFERÊNCIAS BIBLIOGRÁFICAS}

ADAMS, J.P.; ROUSSEAU, R.J.; ADAMS, J.C. 2007. Genetic performance and maximizing genetic gain through direct and indirect selection in cherrybark oak. Silvae Genetica, v. 56, n. 2.

CAMARGO, F.F.; $\quad$ COSTA,R.B.; RESENDE, M.D.V.; ROA, R.A.R.; SANTOS, L.V.; FREITAS, A.C.A. 2010. Variabilidade genética para caracteres morfométricos de matrizes de castanhado-brasil da Amazônia Matogrossense. Acta Amazonica, v.40, n.4, 705-710. 
COSTA, R.B.; RESENDE, M.D.V.; ROA, R.A.R. ; BUNGENSTAB, D.J.; MARTINS, W.J.; ROEL, A.R. 2009. Melhoramento genético de erva-mate nativa do Estado de Mato Grosso do Sul. Bragantia, v. 68, n.3, p. 611-619.

COSTA, R.B.; RESENDE, M.D.V.; CONTINI, A.Z.; REGO, F.L.H.; ROA, R.A.R.; MARTINS, W,J. 2005. Avaliação genética dentro de indivíduos de erva-mate (Ilex paraguariensis St. Hil.), na região de Caarapó, MS, pelo procedimento REML/BLUP. Ciência Florestal, Santa Maria, v.15, n.4, p.371376.

FARIAS NETO, J.T.; RESENDE, M.D.V. 2001. Aplicação da metodologia de modelos mistos (Reml/Blup) na estimação de componentes de variância e predição de valores genéticos em pupunheira (Bactris gasipaes). Revista Brasileira de Fruticultura, v.23,n.2, p.320-324.

FERREIRA, A.G.; KASPARY, R.; FERREIRA, H.B.; ROSA, L.M. 1983. Proporção de sexo e polinização em Ilex paraguariensis St. Hil. Brasil Florestal, n.53, p. 29-33.

FURLANI, R.C.M.; MORAES, M.L.T.; RESENDE, M.D.V.; FURLANI JUNIOR, E.; GONÇALVES, P.S.; VALÉRIO FILHO, W.V.; PAIVA, J.R. 2005. Estimation of variance components and prediction of values in rubber tree breeding using the Reml/Blup procedure. Genetics and Molecular Biology, v.28, n.2, p.271-276.

GONÇALVES, P.S.; MORAES, M.L.T.; BORTOLETTO, N.; COSTA, R.B.; GONÇALVES, E.C.P. 2005. Genetic variation in growth traits rubber trees (Hevea brasiliensis) growing in the
Brazilian State of São Paulo. Genetics and Molecular Biology, v.28, n.4, p.765772 .

IBGE. Produção da extração vegetal e da silvicultura em 2009. 2010. Rio de Janeiro: Instituto Brasileiro de Geografia e Estatística, v. 24, p. 1-45.

MISSIO, R.F.; SILVA, A.M.; DIAS, L.A.S.; MORAES, M.L.T.; RESENDE, M.D.V. 2005. Estimates of genetic parameters and prediction of additive genetic values in Pinus kesya progenies. Crop Breeding and Applied Biotechnology, v.5, p.394401.

POTT, A.; POTT, V. Espécies de fragmentos florestais em Mato Grosso do Sul. 2003. In: COSTA, R.B. (Org.). Fragmentação florestal e alternativa de desenvolvimento rural na região Centro-Oeste. Campo Grande: UCDB, p. 26-52.

RESENDE, M.D.V. 2002a. Software Selegem-REML/BLUP. Colombo: Embrapa Florestas, 67p.

RESENDE, M.D.V. 2002b. Genética biométrica e estatística no melhoramento de plantas perenes. Brasília: Embrapa Informação Tecnológica: Colombo: Embrapa Florestas, 975p.

ROSSE, L.N.; FERNANDES, J.S.C. 2002. Escolha de caracteres para o melhoramento genético em erva-mate por meio de técnicas multivariadas. Ciência Florestal, v.12, n.1, p.21-27.

SCHERER, R. A. 1997. Early selection of yerba mate (Ilex paraguariensis St. Hil.) in Argentina. Bonn: Rheinischen Friedrich-Wilhelms- Universitat, 58p. 
SIMEÃO, R.M.; STURION, J.A.; RESENDE, M.D.V. 2002. Avaliação genética em erva-mate pelo procedimento BLUP individual multivariado sob interação genótipo $\mathrm{x}$ ambiente. Pesquisa Agropecuária Brasileira, v.37, n.11, p.1589-1596, nov.

STURION, J.A.; RESENDE, M.D.V. 2010.

Melhoramento genético da erva-mate.

Colombo: Embrapa Florestas, v. 1. 274 p. 